\title{
Modeling detachment physics in the NSTX snowflake divertor
}

\author{
E.T. Meier ${ }^{a, *}$, V.A. Soukhanovskii ${ }^{a}$, R.E. Bell ${ }^{\mathrm{b}}$, A. Diallo ${ }^{\mathrm{b}}$, R. Kaita ${ }^{\mathrm{b}}$, B.P.

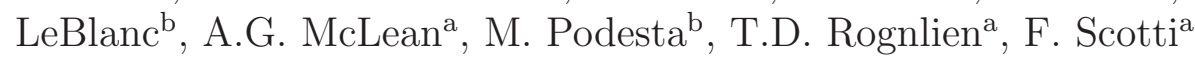 \\ ${ }^{a}$ Lawrence Livermore National Laboratory, Livermore, California 94551, USA \\ ${ }^{b}$ Princeton Plasma Physics Laboratory, Princeton, New Jersey 08540, USA
}

\begin{abstract}
The snowflake divertor is a proposed technique for coping with the tokamak power exhaust problem in next-step experiments and eventually reactors, where extreme power fluxes to material surfaces represent a leading technological and physics challenge. In lithium-conditioned National Spherical Torus Experiment (NSTX) discharges, application of the snowflake divertor typically induced partial outer divertor detachment and severalfold heat flux reduction. UEDGE is used to analyze and compare conventional and snowflake divertor configurations in NSTX. Matching experimental upstream profiles and divertor measurements in the snowflake requires target recycling of 0.97 vs. 0.91 in the conventional case, implying partial saturation of the lithium-based pumping mechanism. Density scans are performed to analyze the mechanisms that facilitate detachment in the snowflake, revealing that increased divertor volume provides most of the parallel heat flux reduction. Also, neutral gas power loss is magnified by the increased wetted area in the
\end{abstract}

\footnotetext{
*Corresponding author

Email address: meier23@llnl.gov (E.T. Meier)
} 
snowflake, and plays a key role in generating volumetric recombination.

Keywords:

NSTX, UEDGE, divertor modeling, divertor geometry, detachment

PACS: 52.55.Rk, 52.55.Fa, 52.65.Kj

\section{Introduction}

In ITER and other next-step magnetic fusion devices, power exhaust will challenge the integrity of divertor plasma-facing components [1]. In spherical tokamaks (STs), power exhaust is especially intense, due to a relatively small divertor footprint [2]. A variety of divertor heat flux mitigation research on the National Spherical Torus Experiment (NSTX) has aimed to contribute to the physics understanding that will be critical for ITER operation, prepare for NSTX Upgrade [3] operation, and support divertor design for next-step STs [4]. Experiments with the snowflake divertor (SFD) configuration are the subject of the modeling analysis presented here.

In the SFD [5], a secondary X-point is introduced near the primary Xpoint, providing heat flux mitigation through a variety of mechanisms [6]. In lithium-conditioned NSTX experiments, the SFD enabled partially detached divertor operation $[7,8]$ without the additional divertor deuterium injection required for such operation in conventional divertor (CD) configurations $[9,10]$. Signatures of strong recombination were observed, including a dramatic increase in divertor $D_{\alpha}$ emission. Peak heat flux was typically reduced severalfold, from $\sim 5$ to $\sim 1 \mathrm{MW} / \mathrm{m}^{2}$.

The NSTX SFD experiments involve the intersection of two challenging areas of fusion physics research — divertor detachment and advanced divertor 
configuration — providing a particularly rich subject for modeling. The UEDGE code $[11,12]$, is used to analyze CD and SFD experimental results. This analysis aims to show the relative performance of the CD and SFD configurations, rather than to provide quantitative information about the configurations individually.

\section{Modeling setup}

UEDGE $[11,12]$ is a $2 \mathrm{D}$ multi-fluid edge transport code, based on the Braginskii two-fluid model [13]. Turbulent perpendicular transport is ap-

proximated with "effective" diffusivities. A neutral fluid model is employed, with a single fluid representing atoms and molecules. Neutral particle fluxes are determined by a Navier-Stokes implementation in the direction parallel to the magnetic field [14], and by a diffusive model in the perpendicular directions, with diffusion mediated by resonant charge exchange (CX) and scattering collisions [12]. Under the assumption of strong ion-neutral coupling via CX, a combined ion-plus-neutral energy equation is employed, and ion and neutral gas temperatures $\left(T_{i}\right.$ and $T_{g}$, respectively) are typically assumed to be equal (as in this work). Two impurity models are available: one uses a fixed impurity concentration, and computes radiation assuming coronal conditions; the other is a charge-state-resolved model that tracks individual charge state densities, determines velocities by force balance, and includes impurity ion energy evolution in the combined ion-plus-neutral energy equation. The research presented here focuses on results with a fixed fraction carbon model. Carbon concentration in the divertor region, where carbon radiation is most important, is unknown; $3 \%$ concentration, in rough agreement with upstream measurements, is chosen. 
NSTX discharge 141240 is modeled at 439 and $905 \mathrm{~ms}$, representing the CD and SFD phases, respectively. The LRDFIT Grad-Shafranov code [15] is used to generate equilibria at these times. Divertor regions of the UEDGE fluxfitted grids are presented in Fig. 1. According to [16], for heat flux mitigation comparable to an exact SFD (which has co-located primary and secondary $\mathrm{X}$-points), the distance between the primary and secondary SFD X-points $\left(d_{X X}\right)$ should be less than $\lambda_{q}^{X}$, which is the heat flux width mapped to the poloidal position of the primary X-point. The heat flux width for discharge 141240 is approximately $5 \mathrm{~mm}$, which maps to $\lambda_{q}^{X}=18 \mathrm{~cm}$. In this SFD LRDFIT equilibrium, $d_{X X}=24 \mathrm{~cm}$, which slightly exceeds $\lambda_{q}^{X}$; nevertheless, significant SFD effects are expected. ${ }^{1,2}$ Compared to the CD, the geometric expansion factor (accounting for both flux expansion and tilt of flux surfaces with respect to the target) for the SFD outer target is increased by $\sim 100 \%$ at the strike point and more than $500 \%$ at a position corresponding to 5 $\mathrm{mm}$ beyond the separatrix at the outer midplane (OMP). The magnetic connection length from the OMP to outer target is increased $\sim 50 \%$ in the SFD. At the inner target, CD and SFD expansion and connection length are nearly identical. The divertor volume, indicated in Fig. 1, is increased from $0.04 \mathrm{~m}^{3}$ in the CD to $0.11 \mathrm{~m}^{3}$ in the SFD. An aspect of SFD magnetic topology that can have negative consequences is that the angle of incidence

\footnotetext{
${ }^{1}$ Configurations with near-target secondary $\mathrm{X}$-points within (or very nearly within) the flux surface corresponding to one heat flux width, but with $d_{X X}>\sim 2 \lambda_{q}^{X}$, would be considered X-divertors [17] rather than SFD.

${ }^{2}$ The version of UEDGE used for the present modeling is not capable of capturing the magnetic topology associated with the secondary X-point of the SFD, and it was necessary to model a configuration with the secondary $\mathrm{X}$-point outside of the plasma volume (below the target in this case). A version of UEDGE capable of modeling the secondary X-point is under development [18].
} 
$(\gamma)$ of the magnetic field on the target is reduced with strong target flux expansion, and is as small as $0.6^{\circ}$ in the SFD depicted in Fig. 1. Small $\gamma$ can result in concentrated heat loads ("hot spots") on slightly misaligned divertor surfaces. Detachment of the plasma in regions with small $\gamma$ reduces the heat flux conducted along field lines and can minimize this problem. In the NSTX SFD experiments, partial detachment occurs, and hot spots are not observed.

Single-null grids, desirable for their computational tractability, are used. In this discharge, the OMP separation of the primary separatrix and the upper X-point separatrix is $7 \mathrm{~mm}$ in the $\mathrm{CD}$ case and and $11 \mathrm{~mm}$ in the SFD, limiting the grids to these relatively small OMP widths. As a result, the boundary conditions (BC) at the outer "walls" can significantly influence the UEDGE solutions.

In the analysis below, UEDGE is used in two modes. In "Mode 1," a range of separatrix shifts $\left(\delta_{\text {sep }}\right)$ and divertor target recycling $\left(R_{\text {div }}\right)$ is explored to find the combination of these two key parameters that provides a fit to NSTX OMP and divertor diagnostic data. ${ }^{3}$ Specifically, Thomson scattering [19] and charge exchange recombination spectroscopy [20] are matched at the OMP, and IR-thermography-based heat flux [21], and line-integrated $\mathrm{D}_{\alpha}$ brightness [22] constrain the divertor solution. To facilitate Mode 1, radial diffusivity profiles are determined using the automated profile fitting procedure introduced by Canik [23]. Mode 1 radial diffusivity profiles are assumed to be poloidally uniform. In "Mode 2," the density at the core-edge

\footnotetext{
${ }^{3} \delta_{\text {sep }}$ refers to a shift of the OMP diagnostic data with respect to the equilibrium and thus with respect to the UEDGE grid.
} 
interface is varied, while holding other settings fixed, to compare CD and SFD physics. Diffusivities in Mode 2 are uniform (radially and poloidally). Both Modes use 3\% carbon concentration. Solutions in Section 3 are all in steady state.

$3 \mathrm{MW}$ input power, corresponding to $4 \mathrm{MW}$ neutral beam power minus assumed $25 \%$ radiation and fast ion losses, is split evenly between ion and electron channels at the core boundary. In Mode 1, ion input from the core matches the neutral beam injection, which is $4.0 \times 10^{20} \mathrm{~s}^{-1}$. In Mode 2, the core density is fixed. At the outer wall, in Mode 1, density and temperatures are fixed as needed to match the OMP diagnostic data; in Mode 2, 2-cm gradient scale length BC are employed for these quantities. In Mode 1, density is set to a single value along the wall, but the wall temperatures vary smoothly (as a half cycle of a sinusoid), from maxima at the OMP wall (where measured temperatures are matched) to $10 \mathrm{eV}$ at a poloidal distance of $1.5 \mathrm{~m}$ (in both directions) from the OMP; at greater poloidal distances, temperatures are set to $10 \mathrm{eV}$. (This reduction at locations far from the OMP prevents artificial inflow of energy to the divertor regions.) In both Modes, $1.9 \times 10^{21} \mathrm{~s}^{-1}$ of neutral gas is injected at the inner midplane, representing experimental injection at that location. At the private flux wall, the radial (i.e., perpendicular to flux surfaces) gradients of density and temperatures are set to $2 \mathrm{~cm}$. Outer wall recycling (of both ions and neutrals) is 80 and $90 \%$ for the CD and SFD, respectively. (In the relatively narrow $7-\mathrm{mm}$ CD grid, stronger pumping is needed to prevent excessive neutral density at the OMP.) At the private flux wall, ion recycling is again 80 and $90 \%$ for the CD and SFD, respectively, but neutral recycling is $100 \%$ for both 
configurations. For the targets, 100\% neutral recycling is assumed in both Modes, and ion recycling is discussed in Section 3. Parallel neutral velocity at the targets is set to a fraction ( 0.5 in this work) of the ion parallel velocity, which is set to the plasma sound speed. Heat transmission to the targets is determined by standard sheath theory [24], with electron and ion heat transmission coefficients of 4 and 2.5, respectively.

To improve modeling in the low-temperature detached regime, the divertor target $\mathrm{BC}$ have been modified to include the transfer of power by neutral deuterium gas. Outgoing neutrals deposit most of their energy on the targets, because energy reflection is low, i.e, $<20 \%$ for carbon targets like those in NSTX ([24], Sec. 3.1). Based on the neutral fluid density and temperature, and assuming a Maxwellian distribution, the one-way neutral gas flux to the target is $\Gamma_{g, o w} \equiv 1 / 4 v_{t h, g} n_{g}$, where $v_{t h, g}$ is the neutral gas thermal speed, and $n_{g}$ is the gas density ([24], Eqn. 2.24). The total gas power loss $(\mathrm{GPL})$ is $2 \Gamma_{g, \text { ow }} T_{g}$ ([24], Eqn. 2.30). In addition to this power loss, an effect related to molecular dissociation is taken into account. Though the atomic vs. molecular composition of gas density is unknown in UEDGE, the incident flux, $\Gamma_{g, \text { ow }}$, is assumed to be purely atomic; when a significant ion population is present, this is a reasonable assumption - CX strongly couples atomic neutrals to the main ion flow, driving them toward the target, while molecular gas more freely diffuses away from the targets. Incident deuterium atoms are largely $(>80 \%)$ thermalized to the target material temperature and converted to deuterium molecules ([24], Sec. 3.1). To complete the cycle, the exiting molecular gas is dissociated and returns as $\Gamma_{g, \text { ow }}$. The most energetic dissociation process is the transfer of the Franck-Condon energy 
$\left(\varepsilon_{F C} \equiv 5 \mathrm{eV}\right.$ per atom) from electrons to dissociated atoms. This process, though it occurs volumetrically, is accounted for by using the target $\mathrm{BC}$ to transfer energy from the electron species to the ion species. The total GPL heat fluxes (with units of power per unit area) to the target for ion-plusneutral and electron fluids are, respectively, $q_{G P L}^{i+n}=2 \Gamma_{g, o w} T_{g}-\Gamma_{g, o w} \varepsilon_{F C}$, and $q_{G P L}^{e}=\Gamma_{g, o w} \varepsilon_{F C}$. The heat flux $2 \Gamma_{g, o w} T_{g}$ is always positive, i.e., depositing heat on the target. The energy transfer term $\Gamma_{g, o w} \varepsilon_{F C}$ is also always positive, thus adding energy to the interior ion-plus-neutral fluid, and extracting energy from the electron fluid.

The UEDGE fluid neutral model (including this GPL treatment), represents a significant simplification of actual divertor gas kinetics, so results using the model should be seen as qualitative and exploratory rather than quantitative. More accurate modeling could be achieved by applying a Monte Carlo neutral model (such as EIRENE [25]), or by employing a more accurate fluid model with an independent neutral temperature [26] and perhaps with an entirely separate molecular fluid.

\section{Modeling results and discussion}

As presented in Fig. 2, a scan of the $\delta_{\text {sep }}$ vs. $R_{\text {div }}$ parameter space (using Mode 1 operation) shows a best-fit ${ }^{4} \mathrm{CD}$ simulation at $R_{d i v}=0.91$ and $\delta_{\text {sep }}=2.1$ $\mathrm{cm}$. In this scan and the SFD scan discussed below, the range of $\delta_{\text {sep }}$ from 1.7 to $2.2 \mathrm{~cm}$ corresponds to OMP electron separatrix temperatures $\left(T_{e}^{\text {sep }}\right)$ from 52 to $82 \mathrm{eV}$. Fig. 2 also shows divertor profiles of heat flux and $D_{\alpha}$

\footnotetext{
${ }^{4}$ The best fit is determined by matching the peak values of measured heat flux and $D_{\alpha}$ brightness at the outer divertor target. The reason for this choice is that heat flux data is not available at the inner target.
} 
brightness for the best-fit simulation. The radial locations of the $D_{\alpha}$ data points indicate where the chords of the $D_{\alpha}$ diagnostics (experimental and synthetic) cross the outer target plane at $Z=0.14 \mathrm{~m}$; the chords originate at $R=1.1 \mathrm{~m}$ and $Z=3.5 \mathrm{~m}$. The shortfall seen in the "tail" of the simulated heat flux could be related to the artificial outer wall boundary, which tends to extract power from the outer SOL. The low simulated $D_{\alpha}$ brightness in the inner divertor region $(R \approx 0.23 \mathrm{~m})$ could be caused by the close proximity of the artificial private flux wall, or the lack of drift physics in the modeling. GPL terms have minimal impact in the CD, modifying peak heat flux and $\mathrm{D}_{\alpha}$ brightness results by $<0.5 \%$ in the best-fit case.

In a similar SFD scan presented in Fig. 3 , at $\delta_{\text {sep }}=1.8 \mathrm{~cm}$ and $R_{d i v}=0.97$, maximum outer divertor $\mathrm{D}_{\alpha}$ brightness reaches $5 \times 10^{22} \mathrm{ph} / \mathrm{m}^{2} / \mathrm{s}$, comparable to the experimentally measured brightness. Without GPL terms described in Section 2, the heat flux in the $\delta_{\text {sep }}$ vs. $R_{\text {div }}$ space is similar to that shown in Fig. 3, but $D_{\alpha}$ brightness remains $<1 \times 10^{22} \mathrm{ph} / \mathrm{m}^{2} / \mathrm{s}$. As shown in the radial profiles, simulated peak $\mathrm{D}_{\alpha}$ brightness and peak heat flux are similar to the experimental peaks, but the profile shapes differ. Disagreement could stem from a variety of factors, including those discussed above in relation to the CD $D_{\alpha}$ profile. Another consideration is that UEDGE neutral model limitations may prevent more precise agreement.

The results shown in Figs. 2 and 3 suggest that $R_{\text {div }}$ in the best-fit SFD is higher than in the CD (0.97 vs. 0.91). Though there is uncertainty due to modeling limitations, there are physical reasons to expect increased recycling in the detached SFD operation. At low target temperatures, low sheath voltage results in low-energy incident ions. These ions are more likely 
to interact only in the upper layers of the target material, contributing to saturation of the deuterium retention mechanism. Also, the neutral particle flux in the SFD is $3-4 \times 10^{24} \mathrm{~m}^{-2} \mathrm{~S}^{-1}$ in the strike point region, compared to $<10^{23}$ in the CD. (For reference, the peak ion fluxes in both SFD and CD cases are $1-2 \times 10^{23} \mathrm{~m}^{-2} \mathrm{~s}^{-1}$.) The chemistry of the NSTX lithium-conditioned graphite targets is complicated, but there is experimental evidence of saturation at NSTX-relevant deuterium ion fluences [27]; elevated neutral deuterium fluence and low-energy ion incidence in the SFD could accelerate such saturation.

Midplane cross-field diffusivity profiles for the best-fit CD and SFD simulations of Figs. 2 and 3 are comparable to those found by Canik for a lithium-conditioned H-mode NSTX discharge [23]. Particle diffusivities are similar for the CD and SFD, rising roughly linearly from $0.2 \mathrm{~m}^{2} / \mathrm{s}$ at $1 \mathrm{~cm}$ inside the separatrix to $0.4-0.5 \mathrm{~m}^{2} / \mathrm{s}$ at $0.5 \mathrm{~cm}$ beyond the separatrix. Electron thermal diffusivites are $5 \mathrm{~m}^{2} / \mathrm{s}$ at $1 \mathrm{~cm}$ inside the separatrix in both cases, rise to 13 and $10 \mathrm{~m}^{2} / \mathrm{s}$ (for the $\mathrm{CD}$ and $\mathrm{SFD}$, respectively) at the separatrix, and reach 17 and $25 \mathrm{~m}^{2} / \mathrm{s}$ (for the $\mathrm{CD}$ and $\mathrm{SFD}$, respectively) at $0.5 \mathrm{~cm}$ beyond the separatrix. Ion thermal diffusivities (to which divertor solutions are relatively insensitive compared to electron thermal diffusivities) range from $5-10 \mathrm{~m}^{2} / \mathrm{s}$ in the region from $1 \mathrm{~cm}$ inside to $0.5 \mathrm{~cm}$ outside the separatrix.

Directly comparing the CD and SFD in a scan of core density $\left(n_{\text {core }}\right)$ (i.e., Mode 2 operation) reveals the mechanisms by which SFD geometry enables partial detachment. Target recycling is $95 \%$. Uniform particle and thermal diffusivities, $D=0.4 \mathrm{~m}^{2} / \mathrm{s}$, and $\chi_{i, e}=10 \mathrm{~m}^{2} / \mathrm{s}$, similar to the values seen near the separatrix in the fitted solutions described above, are used. Results are 
summarized in Fig. 4. For both CD and SFD, panels (a)-(e) show evidence of partial detachment: several-hundred-fold increase in outer strike point (OSP) neutral gas denities, sub-eV OSP electron temperatures, reduced heat flux, increased radiation, and "rollover" in target particle flux. In the CD, detachment onset occurs gradually between $n_{\text {core }}=3-4 \times 10^{19} \mathrm{~m}^{-3}$, while in the SFD, onset occurs sharply at $n_{\text {core }}=2.6-2.8 \times 10^{19} \mathrm{~m}^{-3}$. The roles of three key features of SFD geometry can be identified: 1) Increased connection length $\left(L_{C}\right)$. Fig. $4(\mathrm{c})$ shows similar $q_{\|}$until SFD detachment onset, suggesting that perpendicular energy losses are not principally different in the SFD, despite increased $L_{C}$ (which could enable such losses). Through 1D thermal conduction physics (cf., the two-point model [24]), increased $L_{C}$ reduces $T_{e}$ at the target. UEDGE naturally captures this effect, which is estimated to yield $20 \%$ lower $T_{e}$ (given $\sim 50 \%$ increase in $L_{C}$ ). 2) Increased divertor volume $\left(V_{\text {div }}\right)$. With similar density, the increased SFD $V_{\text {div }}$ results in a total divertor particle inventory of $2.1 \times 10^{18}$ vs. $1.0 \times 10^{18}$ in the $\mathrm{CD}$. As seen in Fig. 4(d), the radiated power is correspondingly increased by a factor of two. 3) Increased wetted area $\left(A_{\text {wet }}\right)$. Increased SFD $A_{\text {wet }}$ accounts for the lower $q_{\perp}$ in Fig. 4(c) but, more importantly for detachment physics, enables GPL, which increases linearly with $A_{\text {wet }}$. GPL power extraction, shown in Fig. 4(d), contributes to $q_{\|}$reduction, and acts as a trigger for strong recombination and associated partial detachment. GPL is pivotal in reducing $T_{e}^{O S P}$ below $0.5 \mathrm{eV}$, inducing the jump in recombination shown in panel (e) - SFD simulations without GPL show $T_{e}^{O S P}>0.5 \mathrm{eV}$ and a modest rise in recombination to $0.3 \times 10^{22} \mathrm{~s}^{-1}$ at $n_{\text {core }}=3.4 \times 10^{19} \mathrm{~m}^{-3}$. Thus, the modeling shows that the synergistic combination of these three features of 
SFD geometry reduces target $T_{e}$ and $q_{\|}$, and promotes partial detachment at much lower $n_{\text {core }}$ than seen in the CD. Fig. $4(\mathrm{f})$ shows the total divertor plugging efficiency, $\zeta$, defined as the ratio of ionization in the divertor volume to neutral sources, including recombination and recycling. In detachment, $\zeta$ declines as cooler plasma conditions allow neutral leakage. In the fitted SFD, $\zeta=0.86$ - similar to $\zeta$ after detachment in panel (f). In the fitted CD, however, $\zeta=0.73$, due to $\sim 10 \times$ lower divertor density than in the $n_{\text {core }}$ scan.

Modeling with charge-state-resolved carbon has been attempted, but results in $<1 \%$ carbon concentration in the divertor region except in a thin layer within a few mm of the targets, where concentration is $\sim 3 \%$. The resulting carbon radiation is not sufficient to produce detachment. The reasons for this must be unfolded in future research, but a prime culprit is the lack of plasma drift physics in the modeling. For example, estimating the OSP poloidal $E \times B$ drift velocity as $v_{E x B}^{\text {pol. }}=3 T_{e} /\left(\lambda_{T e} B_{t o r .}\right)$ [24], and given $T_{e} \approx$ $20 \mathrm{eV}, \lambda_{T e} \approx-0.05 \mathrm{~m}$ (negative because $T_{e}$ typically increases radially at the OSP), and $B=1.2 \mathrm{~T}$, gives $v_{E x B}^{\text {pol. }}=-1000 \mathrm{~m} / \mathrm{s}$ (away from the target). This speed is comparable to the poloidal component of the ion sound speed, which is $1500 \mathrm{~m} / \mathrm{s}$ for $T_{e, i} \approx 20 \mathrm{eV}$ at $\sim 2$-degree incidence. This drift effect would likely reduce the divertor impurity "trapping" effect, in which friction with deuterium ion flow concentrates impurity ions (i.e, carbon ions in this NSTX case) near divertor targets [24, 28], and result in higher upstream carbon concentration as required for detachment. ${ }^{5}$

\footnotetext{
${ }^{5}$ Drift effects do not enter the momentum equation as forces; thus, a simple estimate of the effect of the poloidal $E \times B$ drift using force balance is not possible. To analyze the effects of plasma drifts, a full set of edge transport equations with drifts $[29,30]$ should be solved self-consistently. Recent advances [31] have made such analysis possible in the
} 


\section{Conclusions}

Results from UEDGE operated in a profile-fitting mode to recreate the edge and divertor plasma conditions in NSTX SFD experiments show a significantly higher target recycling in the SFD case: $97 \%$ in the SFD vs. $91 \%$ in the CD. Higher $R_{\text {div }}$ in the SFD could be related to partial saturation of the lithium-conditioned targets.

Using UEDGE in a mode with uniform diffusivities and $R_{d i v}=0.95$, core density is scanned to identify SFD physics effects. The dominant effect is the larger SFD $V_{d i v}$, which doubles divertor radiation, while increased $A_{\text {wet }}$ and $L_{C}$ play supporting roles in reducing target $T_{e}$ and $q_{\|}$, and inducing partial detachment. A gas power loss mechanism has been identified that scales linearly with $A_{\text {wet }}$ and triggers strong recombination and associated high neutral densities that might contribute to the target saturation and high recycling seen in the fitted SFD solution.

For conditioned targets, despite local saturation near the strike point(s), persistent strong pumping elsewhere might act to prevent full detachment, enabling the desired partial detachment. Spatially dependent recycling can be addressed in future experiments and modeling. In divertors without strongly pumping targets, SFD-induced saturation will obviously not be a distinguishing characteristic of the SFD.

SFD modeling might benefit from several extensions of the model used here. For example, including drifts might be important for charge-state re-

challenging H-mode ST (which has especially strong drift flows due to low magnetic fields and steep edge gradients), but analysis has been done only for cases without impurities (e.g, [32]). Similar analysis with the addition of impurities is a challenge to be addressed in future work. 
solved carbon modeling. Also, as underscored by the significant neutral gas effects identified in this work, more complete neutral modeling might be beneficial. Several aspects of the SFD concept are not addressed in this modeling, including instability-driven mixing in the region of weak poloidal field [6], and can be considered in future work.

\section{Acknowledgments}

This work has been performed under the auspices of the U.S. Department of Energy by Lawrence Livermore National Laboratory under Contracts DEAC52-07NA27344 and DE-AC02-09CH11466.

[1] A. Loarte, et al., Nucl. Fusion 47 (2007) S203.

[2] T. K. Gray, et al., J. Nucl. Mater. 415 (2011) S360 - S364.

[3] J. E. Menard, et al., Nucl. Fusion 52 (2012) 083015.

[4] J. E. Menard, et al., Nucl. Fusion 51 (2011) 103014.

[5] D. D. Ryutov, Phys. Plasmas 14 (2007) 064502.

[6] D. D. Ryutov, et al., Plasma Phys. Contr. Fusion 54 (2012) 124050.

[7] V. A. Soukhanovskii, et al., Nucl. Fusion 51 (2011) 012001.

[8] V. A. Soukhanovskii, et al., Phys. Plasmas 19 (2012) 082504.

[9] V. A. Soukhanovskii, et al., Phys. Plasmas 16 (2009) 022501.

[10] V. A. Soukhanovskii, et al., Nucl. Fusion 49 (2009) 095025.

[11] T. D. Rognlien, et al., J. Nucl. Mater. 196 (1992) 347 - 351. 
[12] T. D. Rognlien, M. E. Rensink, Fusion Eng. Design 60 (2002) 497 - 514.

[13] S. I. Braginskii, Transport processes in a plasma, in: M. A. Leontovitch (Ed.), Rev. Plasma Phys., Vol. 1, Consultants Bureau, New York, NY, 1965, pp. $205-311$.

[14] F. Wising, et al., Contrib. Plasma. Phys. 36 (1996) 309 - 313.

[15] LRDFIT software, http://nstx-u.pppl.gov/software/lrdfit.

[16] D. D. Ryutov, et al., Contrib. Plasma Phys. 52 (2012) 539 - 543.

[17] M. Kotschenreuther, P. M. Valanju, S. M. Mahajan, J. C. Wiley, On heat loading, novel divertors, and fusion reactors, Phys. Plasmas 14 (2007) 072502.

[18] T. D. Rognlien et al., 56th Annual Meeting of the APS-DPP, New Orleans, LA (2014).

[19] B. P. LeBlanc, et al., Rev. Sci. Instrum 74 (3) (2003) 1659 - 1662.

[20] R. E. Bell, Rev. Sci. Instrum 77 (10).

[21] A. G. McLean, et al., Rev. Sci. Instrum. 83 (2012) 053706.

[22] V. A. Soukhanovskii, et al., Rev. Sci. Instrum 74 (3) (2003) 2094 - 2097.

[23] J. M. Canik, et al., J. Nucl. Mater. 415 (2011) S409 - S412.

[24] P. C. Stangeby, The plasma boundary of magnetic fusion devices, IOP publishing Ltd., 2000.

[25] D. Reiter, et al., J. Nucl. Mater. 241. 
[26] E. T. Meier, U. Shumlak, Phys. Plasmas 19.

[27] C. N. Taylor, et al., J. Nucl. Mater. 415 (2011) S777 - S780.

[28] F. Scotti, Ph.D. thesis, Princeton University (2014).

[29] T. D. Rognlien, D. D. Ryutov, N. Mattor, G. D. Porter, Phys. Plasmas 6 (1999) $1851-1857$.

[30] A. V. Chankin, J. Nucl. Mater. 241.

[31] V. Rozhansky, et al., Nucl. Fusion 49.

[32] V. Rozhansky, et al., Plasma Phys. Control. Fusion 55.

\section{List of Figures}

1 Divertor region of UEDGE grids for conventional divertor (CD) and snowflake divertor (SFD) simulations. Primary X-points are shown with black X's. The red X indicates the SFD secondary X-point. Divertor region control volumes used in the analysis are indicated with dashed lines. For the SFD, the secondary separatrix is indicated with a red dot-dashed line. . 18

2 Conventional divertor $\delta_{\text {sep }}$ vs. $R_{\text {div }}$ scan results. At left, maximum outer divertor heat flux and $D_{\alpha}$ brightness are plotted in the $\delta_{\text {sep }}$ vs. $R_{\text {div }}$ space. Approximate contours of the experimental data for heat flux and $D_{\alpha}$ brightness are shown by the solid and dashed lines, respectively. For the simulation at the intersection of the two contours at $R_{d i v}=0.91$ and $\delta_{\text {sep }}=2.1$ $\mathrm{cm}$, heat flux and $D_{\alpha}$ brightness profiles are shown at right. . 18 
3 Snowflake divertor $\delta_{\text {sep }}$ vs. $R_{\text {div }}$ scan results. At left, maximum outer divertor heat flux and $D_{\alpha}$ brightness are plotted in the $\delta_{s e p}$ vs. $R_{d i v}$ space. For the simulation at $R_{d i v}=0.97$ and $\delta_{\text {sep }}=1.8 \mathrm{~cm}$, heat flux and $D_{\alpha}$ brightness profiles are shown at right. . . . . . . . . . . . . . . . . . 19

4 Results from scan of core density $\left(n_{\text {core }}\right)$ for CD (blue) and SFD (red): (a) ion density at the outer midplane (OMP) separatrix, and ion and neutral gas density at the outer strike point (OSP); (b) electron temperatures at the OSP and OMP; (c) peak parallel and perpendicular incident heat fluxes; (d) total power incident on the outer divertor target, the gas power loss (GPL) component of incident power, and the power radiated in the outer divertor volume; (e) ion flux to the outer target, and volumetric recombination; and (f) total divertor plugging efficiency. . . . . . . . . . . . . . . . . . 20 20 

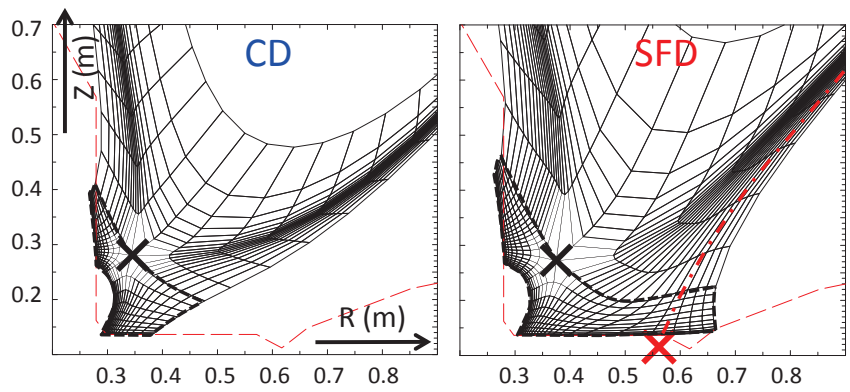

Figure 1: Divertor region of UEDGE grids for conventional divertor (CD) and snowflake divertor (SFD) simulations. Primary X-points are shown with black X's. The red X indicates the SFD secondary X-point. Divertor region control volumes used in the analysis are indicated with dashed lines. For the SFD, the secondary separatrix is indicated with a red dot-dashed line.
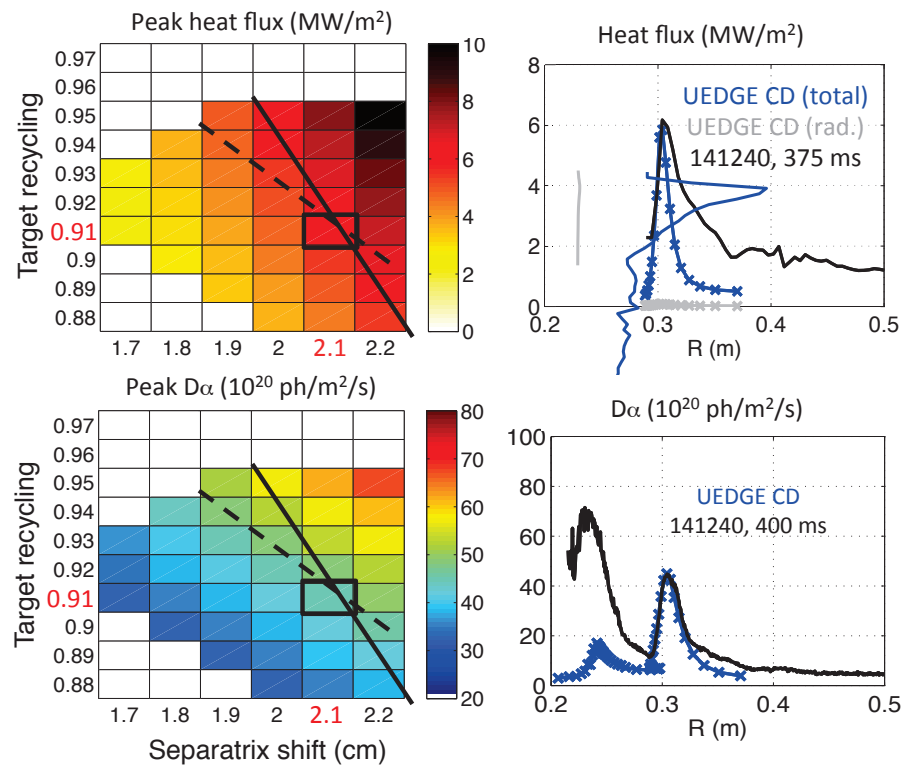

Figure 2: Conventional divertor $\delta_{\text {sep }}$ vs. $R_{\text {div }}$ scan results. At left, maximum outer divertor heat flux and $D_{\alpha}$ brightness are plotted in the $\delta_{\text {sep }}$ vs. $R_{\text {div }}$ space. Approximate contours of the experimental data for heat flux and $D_{\alpha}$ brightness are shown by the solid and dashed lines, respectively. For the simulation at the intersection of the two contours at $R_{d i v}=0.91$ and $\delta_{\text {sep }}=2.1 \mathrm{~cm}$, heat flux and $D_{\alpha}$ brightness profiles are shown at right. 

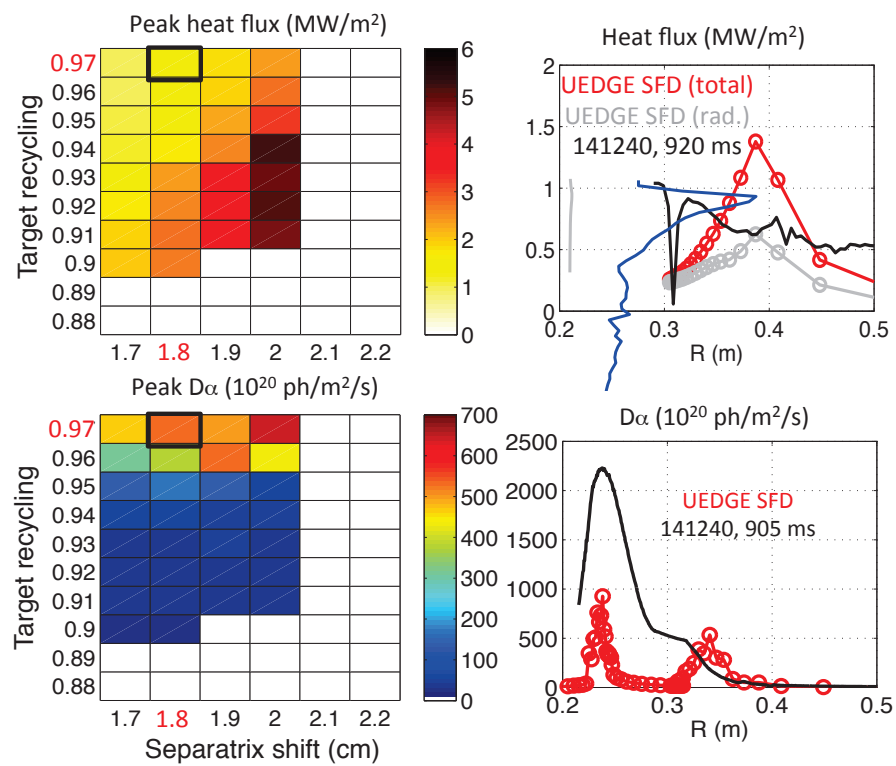

Figure 3: Snowflake divertor $\delta_{\text {sep }}$ vs. $R_{d i v}$ scan results. At left, maximum outer divertor heat flux and $D_{\alpha}$ brightness are plotted in the $\delta_{s e p}$ vs. $R_{d i v}$ space. For the simulation at $R_{d i v}=0.97$ and $\delta_{s e p}=1.8 \mathrm{~cm}$, heat flux and $D_{\alpha}$ brightness profiles are shown at right. 

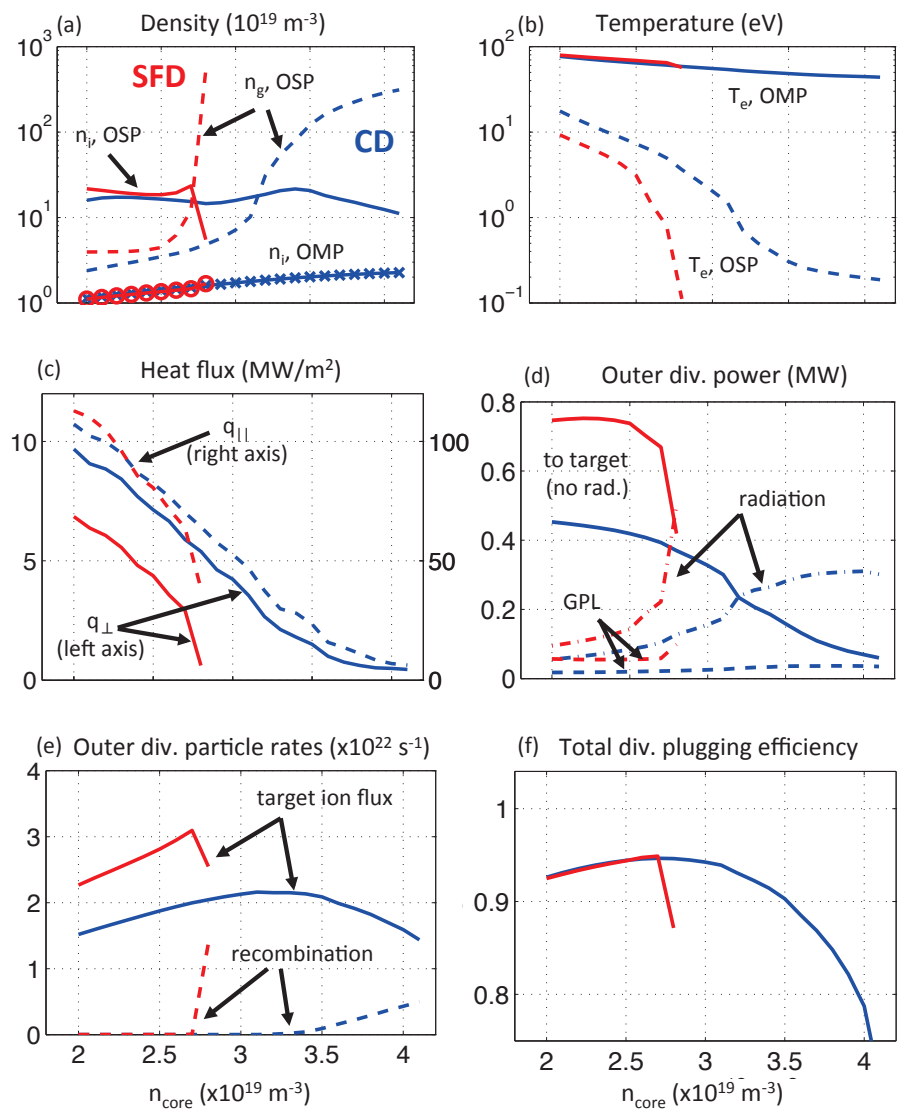

Figure 4: Results from scan of core density $\left(n_{\text {core }}\right)$ for CD (blue) and SFD (red): (a) ion density at the outer midplane (OMP) separatrix, and ion and neutral gas density at the outer strike point (OSP); (b) electron temperatures at the OSP and OMP; (c) peak parallel and perpendicular incident heat fluxes; (d) total power incident on the outer divertor target, the gas power loss (GPL) component of incident power, and the power radiated in the outer divertor volume; (e) ion flux to the outer target, and volumetric recombination; and (f) total divertor plugging efficiency. 